\title{
Quantum Delocalization of Molecular Hydrogen in Alkali-Graphite Intercalates
}

\author{
Arthur Lovell, ${ }^{1, *}$ Felix Fernandez-Alonso, ${ }^{1,+}$ Neal T. Skipper, ${ }^{2}$ Keith Refson, ${ }^{3}$ \\ Stephen M. Bennington, ${ }^{1, \$}$ and Stewart F. Parker ${ }^{1}$ \\ ${ }^{1}$ ISIS Facility, Rutherford Appleton Laboratory, Chilton, Didcot, Oxfordshire OX11 OQX, United Kingdom \\ ${ }^{2}$ Department of Physics and Astronomy, University College London, Gower Street, London, WC1E 6BT, United Kingdom \\ ${ }^{3}$ Rutherford Appleton Laboratory, Chilton, Didcot, Oxfordshire OX11 OQX, United Kingdom
}

(Received 7 March 2008; published 18 September 2008)

\begin{abstract}
The adsorption of molecular hydrogen $\left(\mathrm{H}_{2}\right)$ in the graphite intercalation compound $\mathrm{KC}_{24}$ is studied both experimentally and theoretically. High-resolution inelastic neutron data show spectral features consistent with a strong pinning of $\mathrm{H}_{2}$ along a single axis. First-principles calculations provide novel insight into the nature of $\mathrm{H}_{2}$ binding in intercalates but fail to account for the symmetry of the $\mathrm{H}_{2}$ orientational potential deduced from experiment. The above discrepancy disappears once the $\mathrm{H}_{2}$ center of mass is allowed to delocalize in the quantum-mechanical sense across three vicinal adsorption sites, naturally leading to the well-known saturation coverage of $\sim 2 \mathrm{H}_{2}$ per metal atom in this material. Our results demonstrate that $\mathrm{H}_{2}$ storage in metal-doped carbon substrates can be severely affected by hitherto unexplored quantummechanical effects.
\end{abstract}

DOI: 10.1103/PhysRevLett.101.126101

PACS numbers: 68.43.Bc, 61.05.F-, 71.15.Mb

The efficient storage of hydrogen represents an important prerequisite for a sustainable, low- $\mathrm{CO}_{2}$ economy [1]. Owing to their light weight and large surface areas, carbonbased nanostructured materials (CNMs) have been extensively studied as candidates to meet the US-DOE 2010 target of $6.5 \mathrm{wt} \%$ [1,2]. To date, however, no carbon host has been found to meet such stringent requirements. The feeble interaction between molecular hydrogen $\left(\mathrm{H}_{2}\right)$ and graphitic carbon is not likely to be sufficient for roomtemperature applications [3], yet recent work on carbon nanohorns [4,5] and nanoscrolls [6] report an appreciable enhancement of $\mathrm{H}_{2}-\mathrm{CNM}$ interactions solely via geometric confinement. In view of the above, the introduction of suitable dopants (particularly metals) into CNMs represents a viable way forward to enhance sorption energies. Whereas abundant theoretical work exists to support these considerations [6,7], experiments probing $\mathrm{H}_{2}$ binding in metal-doped CNMs are scarce. Careful experiments by Yang [8] have shown a $2 \%$ weight uptake by Li-doped nanotubes. More recent studies [9] on alkali-doped CNM's also report an enhancement of $\mathrm{H}_{2}$ adsorption compared to the undoped species but the microscopic nature of $\mathrm{H}_{2}$ binding sites remains largely unexplored.

Graphite intercalation compounds (GICs) [10] are ideal testbeds to explore $\mathrm{H}_{2}$ storage in metal-doped CNMs. Alkalis readily intercalate into graphite to form lamellar structures of regular and tunable lattice spacing, with a $c$-axis superlattice in which $n$ graphite layers separate each guest layer (so-called stage- $n$ GICs). In particular, the series of stage- 2 compounds of stoichiometry $M \mathrm{C}_{24}(M=$ $\mathrm{K}, \mathrm{Rb}, \mathrm{Cs})$ are known to adsorb $\mathrm{H}_{2}$ up to $\sim 2 \mathrm{H}_{2} / M$ [11]. At temperatures below $100 \mathrm{~K}, M \mathrm{C}_{24}$ adopts a commensurate $\sqrt{7} \times \sqrt{7} M$ superlattice bounded by a higher metal density in the interdomain regions. $\mathrm{H}_{2}$ uptake by $\mathrm{RbC}_{24}[12,13]$ and $\mathrm{CsC}_{24}$ [14] has been investigated by inelastic neutron scattering (INS). INS features were attributed to the presence of two distinct adsorption sites. Our recent work on $\mathrm{KC}_{24}\left(\mathrm{H}_{2}\right)_{x}$ shows no preferential site occupancy for $x=$ 1.0 and $1.5[15,16]$, where $x$ represents the amount of adsorbed $\mathrm{H}_{2}$. This finding strongly suggests the existence of a single adsorption site in $\mathrm{KC}_{24}$, making it a superb candidate for more detailed studies. The aim of this Letter is to probe the $\mathrm{H}_{2}-\mathrm{KC}_{24}$ potential energy landscape via a detailed analysis of high-resolution INS data. The experimental results are supplemented by plane-wave densityfunctional-theory (PW-DFT) calculations so as to provide novel insight into the nature of $\mathrm{H}_{2}$-GIC complexes.

High-purity $\mathrm{KC}_{24}$ was synthesized from nuclear-grade Papyex [17] using a modified one-zone method with a 20 wt \% stoichiometric $\mathrm{K}$ excess at $300{ }^{\circ} \mathrm{C}$ [16]. The sample was annealed for 3 days until the appearance of a uniform blue color signaling charge transfer from the alkali to graphite, and then stored under argon. Neutron measurements were carried out under in situ $\mathrm{H}_{2}$ loading on the IRIS [18] and TOSCA [19] spectrometers. Data were collected at $1.5 \mathrm{~K}$ (IRIS) and $12.5 \mathrm{~K}$ (TOSCA) for $x=0,0.25,0.5$, 1.0 , and 2.0, as measured volumetrically during dosing. An additional over-saturation concentration of $x=6.25$ was measured on IRIS only. The $x=0$ data were used as background spectrum for subsequent subtraction.

Figure 1(a) shows the dependence of the (003) Bragg reflection with $x$. These data were collected following the relaxation of normal $-\mathrm{H}_{2}$ to the para ground state over a period of several hours. The intensity of the $\mathrm{KC}_{24}$ peak at $2.90 \AA$ diminishes with $x$ while a second feature appears above $2.97 \AA$, with its peak maximum shifting steadily towards higher $d$-spacings up to $x=2.0$. Both peaks remain discrete and coexist at all explored $x$, indicating the 

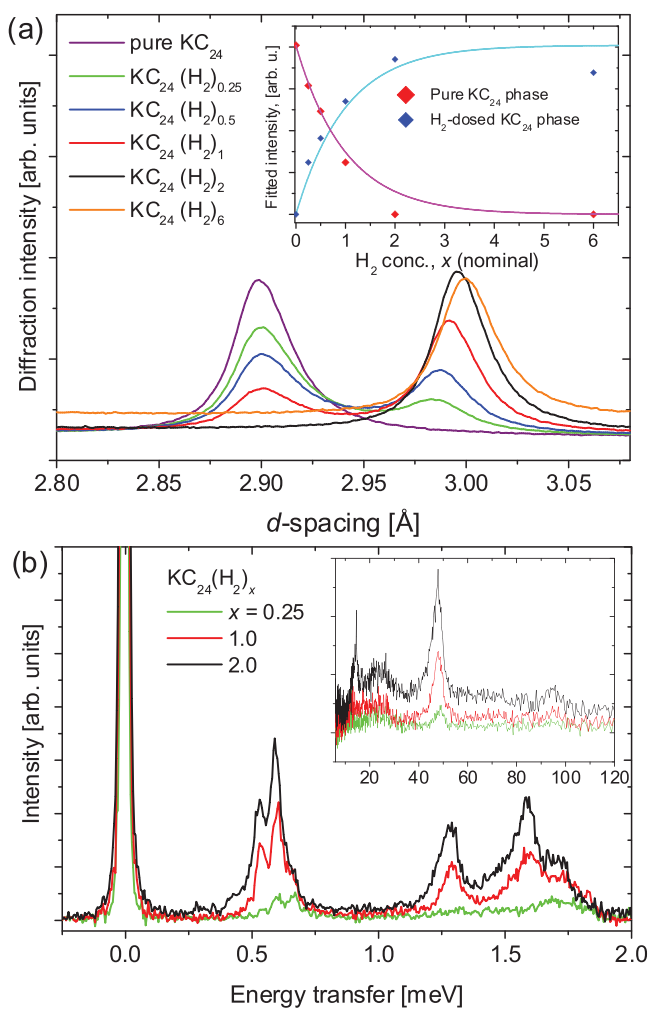

FIG. 1 (color). (a) Evolution of the (003) peak with $x$ as measured on IRIS. The inset shows integrated intensities (symbols) and accompanying fits (lines) using the model described in the text; (b) shows the INS data from IRIS and TOSCA (inset).

presence of domains of pure and hydrogenated $\mathrm{KC}_{24}$. The hydrogenated phase is characterized by up to a $3.3 \%$ expansion of the GIC galleries, from $5.35 \pm 0.01 \AA$ to $5.64 \pm 0.01 \AA$ at $x=2$. At $x=6$, there is a sudden increase in background arising from incoherent scattering of unconverted ortho- $\mathrm{H}_{2}$. There is no significant shift of the higher-lying diffraction peak for $x>2$, indicative of no further site adsorption. The incoherent ortho contribution is thus a symptom of the saturation of the binding sites where ortho-para conversion is allowed to take place [16]. Single-site adsorption with a coverage-independent binding energy obeys an exponential dependence of the form $e^{-x}$ and $\left(1-e^{-x}\right)$ for vacant and filled sites, respectively. As shown in Fig. 1(a), such a dependence is closely followed by our diffraction data.

Figure 1 also shows INS spectra vs $x$. All distinct spectral features display the same $x$ dependence, in line with the diffraction data. At low energies, there are two sets of excitations centered at 0.6 and $1.5 \mathrm{meV}$. Neutron scattering from para $-\mathrm{H}_{2}$ can only be incoherent provided that the total nuclear spin changes upon scattering, leading to a dominant response at and above the lowest para $\rightarrow$ ortho transition [20]. In bulk para- $\mathrm{H}_{2}$, this transition energy is at the free-rotor value of $14.7 \mathrm{meV}$, whereas in $\mathrm{KC}_{24}$ this bandhead has shifted down to $0.6 \mathrm{meV}$, signaling a strong hindering of $\mathrm{H}_{2}$ rotations. Quantitative estimates of the hindering potential can be obtained by considering the
$M$-level splitting of $\mathrm{H}_{2}$ rotational eigenstates in the presence of a potential $V(\Theta, \phi)$ [21]. To lowest order, $V(\Theta, \phi)=V_{\Theta} \sin ^{2} \Theta$, where $\Theta$ and $\phi$ are spherical polar coordinates describing the orientation of $\mathrm{H}_{2}$ relative to the quantization axis. Using the procedure of Ref. [5], a bandhead at $0.6 \mathrm{meV}$ corresponds to $V_{\Theta}=137 \mathrm{meV}$. The upper state of this INS transition correlates with the singly degenerate $|10\rangle$ level in the free-rotor basis, thereby constraining $\mathrm{H}_{2}$ to lie preferentially along the quantization axis. The $V_{\Theta}$ value found for $\mathrm{KC}_{24}$ is $\sim 100$ times higher than in nanotubes [22] and nanohorns [5]. Our calculations also predict a doubly degenerate transition at $51 \mathrm{meV}$, in good agreement with the intense INS feature observed at $48.5 \mathrm{meV}$ [Fig. 1(b)]. Moreover, the sudden appearance of the $\mathrm{H}_{2}$ free-rotor peak at $x=2$ marks the saturation of available GIC adsorption sites, in line with with the diffraction data. In the absence of other librational features, both the fine structure at $0.6 \mathrm{meV}$ and the manifold centered at $1.5 \mathrm{meV}$ can solely arise from the simultaneous excitation of $\mathrm{H}_{2}$ rotational and translational modes.

The PW-DFT code CASTEP [23] was used to study the $\mathrm{H}_{2}$-GIC complex in more detail. A 300-eV PW cutoff using Vanderbilt ultrasoft pseudopotentials [24] within the generalized-gradient approximation (Perdew-BurkeErzerhof, PBE, functional) was used throughout. Our methodology was first benchmarked against the $\mathrm{K}^{+}-\mathrm{H}_{2}$ complex. The rationale behind this choice stems from the order-of-magnitude deviations from free-rotor behavior observed in the INS data. Figure 2 evidences a clear preference for a $T$-shaped geometry $\left(\Theta=90^{\circ}\right)$ with a minimum at $R_{\text {eq }}=2.92 \AA$. The first few radial eigenenergies were computed using the Numerov algorithm [25] and yield a zero-point energy $\left(E_{\mathrm{ZPE}}\right)$ of $13.5 \mathrm{meV}$, as well as a dissociation energy of $E_{D}=78 \mathrm{meV}$. These values are in good agreement with those of Vitillo et al.

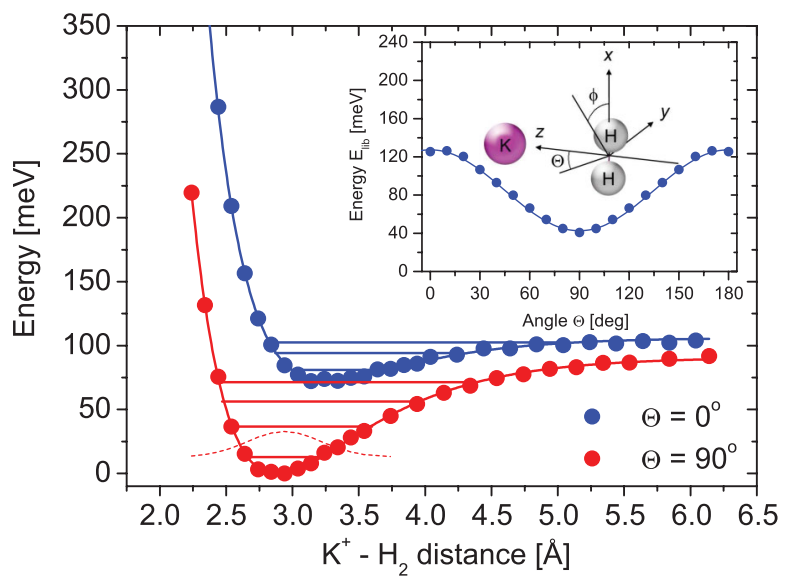

FIG. 2 (color). $\mathrm{K}^{+}-\mathrm{H}_{2}$ potential energy curves. The cartoon defines radial $(R)$ and angular $(\Theta, \phi)$ variables. The inset shows the angular potential at $R_{\text {eq }}$; symbols correspond to PW-DFT calculations whereas the solid line corresponds to the long-range expansion described in the text (quadrupole and polarizability values taken from Ref. [31]). 
$\left(R_{\text {eq }}=2.92-2.94 \AA ; E_{D}=68-78 \mathrm{meV}\right)$ [26]. $T$-shaped and linear geometries are separated by $85 \mathrm{meV}$, an energy approaching the value of $V_{\Theta}$ inferred from the INS data. However, the predicted INS spectrum is characterized by a doubly degenerate bandhead at $9.6 \mathrm{meV}$ reflecting a reversal of $M$-level splittings in favor of $\mathrm{H}_{2}$ alignment normal to $Z$. From the long-range expansion of the angular potential [27], the two dominant interactions are: (a) ionquadrupole, of the form $\left(Q_{\mathrm{H}_{2}} / R_{\mathrm{eq}}^{3}\right) P_{2}(\cos \Theta)$, where $P_{2}(\cos \Theta)$ is the second Legendre polynomial and $Q_{\mathrm{H}_{2}}$ is the $\mathrm{H}_{2}$ quadrupole moment; and (b) ion-induced-dipole, of the form $-\left(1 / R_{\mathrm{eq}}^{4}\right)\left(A+B P_{2}(\cos \Theta)\right)$, where $A$ and $B$ are related to the $\mathrm{H}_{2}$ polarizability tensor via $A=\alpha / 2$ and $B=\left(\alpha_{\text {par }}-\alpha_{\text {per }}\right) / 3$. As shown in Fig. 2, this long-range expansion provides an excellent description of the PWDFT results. Further, it highlights the dominant role of ionquadrupole interactions in dictating the geometry of the $\mathrm{K}^{+}-\mathrm{H}_{2}$ complex, characteristic of a much stronger interaction with the alkali than that found in undoped graphite [28].

To model the GIC, a $\sqrt{7} \times \sqrt{7} K$ superlattice on a $7 \times$ $8 \times 7 k$-point grid was chosen as representative of the main $\mathrm{H}_{2}$-GIC binding motif. This model structure has a $\mathrm{KC}_{14}$ unit cell, as it omits the empty graphite galleries where $\mathrm{H}_{2}$ does not adsorb. For $\mathrm{KC}_{14}$, PW-DFT rightly predicts the transfer of one electron from the alkali metal to the graphene layers [10]. Following these calculations, $\mathrm{H}_{2}$ was inserted in the unit cell and allowed to relax to the energy minimum [cf. Fig. 3]. Neither the $\mathrm{H}_{2}$ bond distance $(\Delta R / R=+0.003)$ nor its internal frequency of vibration $(\Delta \omega / \omega=-0.018)$ changed appreciably during this pro-
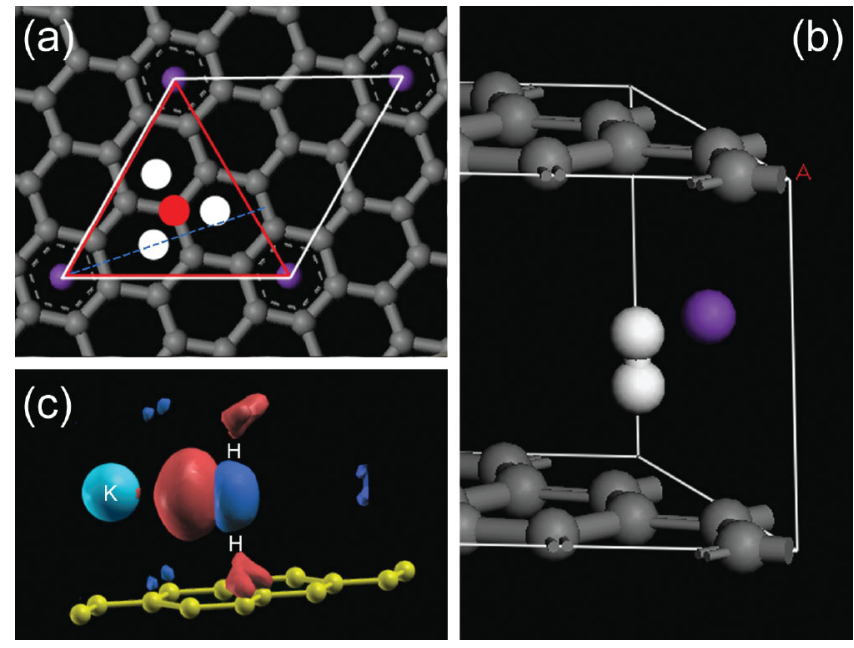

FIG. 3 (color). PW-DFT results: (a) View along the $c$ axis (white lines define the unit cell). The trigonal subunit cell and its center are shown in red. White circles denote three adjacent $\mathrm{H}_{2}$ sites. The blue dashed line shows the direction of the radial energy cut in Fig. 4; (b) side view of the minimum-energy configuration; (c) electron-density-difference map obtained by subtracting the $\mathrm{KC}_{14}$ and $\mathrm{H}_{2}$ densities from that of $\mathrm{KC}_{14} \mathrm{H}_{2}$. Red (blue) denotes electron density gain (loss). cedure. $\mathrm{H}_{2}$ sits $2.87 \AA$ away from the closest $\mathrm{K}$, below the center of the graphite rings. Further, the $\mathrm{H}_{2}$ axis is perpendicular to the graphite layers, adopting a $T$-shaped configuration with respect to the alkali, as in the case of $\mathrm{K}^{+}-\mathrm{H}_{2}$. The electron-density-difference map in Fig. 3(c) shows the distinct appearance of an induced dipole moment on $\mathrm{H}_{2}$ as a result of charge migration towards $K$. Mirroring these changes, there is also charge redistribution in the graphene planes, with negative charge now appearing above and below $\mathrm{H}_{2}$. These findings neatly account for the expansion of the GIC galleries upon the addition of $\mathrm{H}_{2}$, as observed in the diffraction data. $\mathrm{H}_{2}$ radial and angular scans inside the GIC are shown in Fig. 4. For $R<3.5 \AA$, the shape of the $\mathrm{H}_{2}$-GIC curve $\left(E_{\mathrm{ZPE}}=\right.$ $13.9 \mathrm{meV}$ ) is remarkably similar to that of $\mathrm{K}^{+}-\mathrm{H}_{2}$, further reinforcing the notion that the energy landscape around the minimum is dominated by interactions with a single alkali atom. As shown in the inset of Fig. 4, the PWDFT orientational potential $V(\Theta, \phi)$ is adequately described by

$$
V(\Theta, \phi)=V_{\Theta}\left[1-\left(1-\frac{V_{\phi}}{V_{\Theta}} \sin ^{2} \phi\right) \sin ^{2} \Theta\right]
$$

with $V_{\Theta}=126 \mathrm{meV}$ and $V_{\phi}=26 \mathrm{meV}$. The resulting $V(\Theta, \phi)$ is qualitatively similar to that of $\mathrm{K}^{+}-\mathrm{H}_{2}$ except for a small preference for $\mathrm{H}_{2}$ alignment along the GIC $c$-axis as a result of a much weaker interaction with the graphite layers. Although the PBE functional is known to underbind van der Waals complexes, recent work shows that $\mathrm{H}_{2}$-graphite interactions are described with reasonable accuracy [29] and, in particular, rotational barriers agree within several meV with correlated $\operatorname{CCSD}(\mathrm{T})$ methods. The above $V(\Theta, \phi)$ gives rise to a spectral bandhead at $4.71 \mathrm{meV}$, followed by three well-resolved librational transitions at 16,36 , and $38 \mathrm{meV}$, to be contrasted with the single INS feature we observe at $48.5 \mathrm{meV}$. Such a rich spectral progression is the result of a significant departure

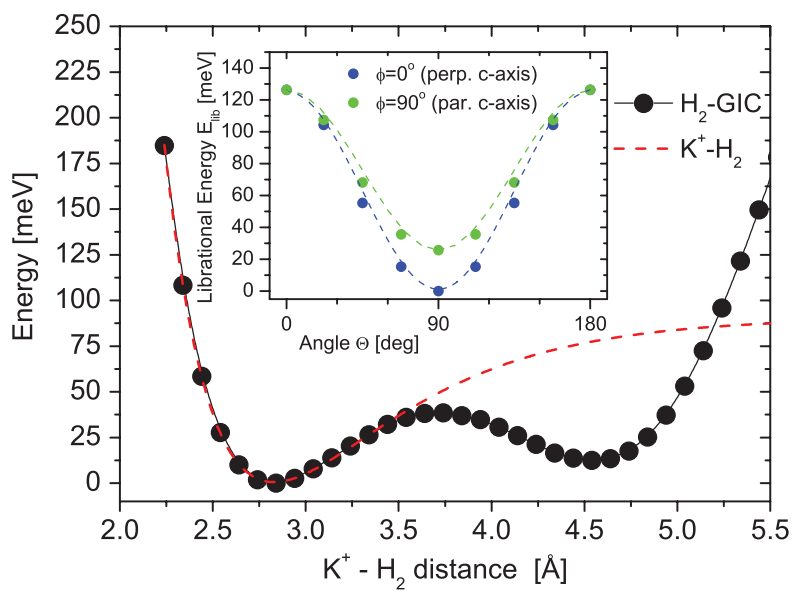

FIG. 4 (color). Radial and angular (inset) scans for the $\mathrm{H}_{2}$-GIC complex. $R, \theta$, and $\phi$ retain the same meaning as in Fig. 2. Dashed lines in the inset correspond to fits using Eq. (1). 
from cylindrical symmetry in the model $\mathrm{H}_{2}$-GIC complex, reflecting sizable energy differences for $\mathrm{H}_{2}$ rotation about the $Z$-axis vs the $X Y$ plane. None of these transitions, however, are observed in the INS data. While PW-DFT provides insightful estimates for $\mathrm{H}_{2}$-GIC interaction energies, it cannot account for the symmetry of the adsorption site. The above discrepancies therefore call for a revision of currently accepted theoretical models.

An underlying assumption in the analysis of the PWDFT results has been $\mathrm{H}_{2}$ localization at a single potential well. Closer inspection of Fig. 3(a) reveals the presence of three neighboring sites located $\sim 1.1 \AA$ away from the center of the trigonal subunit cell. If there is quantummechanical delocalization (QD) of the $\mathrm{H}_{2}$ center of mass across these three sites, the effective orientational potential becomes $V_{\mathrm{QD}}=\frac{1}{3} \sum_{i=1,3} V_{i}\left(\Theta_{i}, \phi_{i}\right)$. To compute $V_{\mathrm{QD}}$, the potential $V\left(\Theta_{i}, \phi_{i}\right)$ at each site [cf. Eq. (1)] is written in terms of spherical harmonics $Y_{\mathrm{JM}}$ to read $V=A+B Y_{20}+$ $C\left(Y_{2+2}+Y_{2-2}\right)$. Using the transformation properties of $Y_{\mathrm{JM}}$ 's [30], a $90^{\circ}$ rotation about $Y$ makes $Z$ coincide with the $c$-axis. $V_{\mathrm{Ri}}(\Theta, \phi)$ then denotes the potential in this new frame and is also of the form $V_{R}=A_{R}+B_{R} Y_{20}+$ $C_{R}\left(Y_{2+2}+Y_{2-2}\right)$. At this point, all three sites can be treated on an equal footing and $V_{\mathrm{OD}}=$ $\frac{1}{3} \sum_{i=1,3} V_{R 1}\left(\Theta_{1}, \phi_{1}+(i-1) \frac{2 \pi}{3}\right)=\langle V\rangle \sin ^{2} \Theta$ with $\langle V\rangle=$ $\frac{1}{2}\left(V_{\Theta}+V_{\phi}\right)$. Thus, QD across three sites of $C_{3}$ symmetry recovers the same functional form as deduced from the INS data. Our PW-DFT calculations yield $\langle V\rangle=76 \mathrm{meV}$. This figure is below the experimental value of $137 \mathrm{meV}$, possibly a consequence of the underestimation of the $\mathrm{H}_{2}$-graphite contribution to the total energy by the PBE funtional [29]. QD of $\mathrm{H}_{2}$ explains why diffraction cannot locate the adsorbant structure and also provides a microscopic mechanism for the maximum uptake of $2 \mathrm{H}_{2} / \mathrm{K}$ in this material. Given the hexagonal symmetry of available adsorption sites surrounding a given alkali, a coverage approaching $6 \mathrm{H}_{2} / \mathrm{K}$ would be expected. QD in the GIC reduces this value to the observed $\sim 2 \mathrm{H}_{2} / \mathrm{K}$. A similar mechanism is likely to be at play in the Cs and Rb GIC analogues but the presence of multiple adsorption sites makes the interpretation of experimental and theoretical data in these systems more involved than in the present case.

In summary, this Letter provides novel insight on $\mathrm{H}_{2}$ physisorption in alkali GICs. QD across several sites is able to reconcile INS data with first-principles calculations in a remarkably holistic manner. The results strongly suggest that quantum-mechanical effects may not be neglected in modeling $\mathrm{H}_{2}$ in CNMs, feasibly extending to all nondissociating hydrogen storage interactions. In particular, QD can reduce the adsorbed $\mathrm{H}_{2}$ density in doped graphites, placing a further limit on the maximal capacity of these storage materials.

We thank RBE Down and CM Goodway from the ISIS User Support Group for their expert assistance and Dr MA
Adams for insightful discussions. AL's doctoral thesis was funded by EPSRC and CCLRC. This work was partially supported by the EU under the NEST FERROCARBON project (CEC 012881).

*A.Lovell@rl.ac.uk
${ }^{+}$F.Fernandez-Alonso@rl.ac.uk

Also at Department of Physics and Astronomy, University College London, Gower Street, London, WC1E 6BT, United Kingdom.

${ }^{\ddagger}$ Also at Department of Physics and Astronomy, University College London, Gower Street, London, WC1E 6BT, United Kingdom.

[1] M. Felderhoff, Phys. Chem. Chem. Phys. 9, 2643 (2007).

[2] A. Züttel, Naturwissenschaften 91, 157 (2004).

[3] R. Ströbel, J. Power Sources 159, 781 (2006).

[4] H. Tanaka et al., J. Am. Chem. Soc. 127, 7511 (2005).

[5] F. Fernandez-Alonso et al., Phys. Rev. Lett. 98, 215503 (2007).

[6] G. Mpourmpakis et al., Nano Lett. 7, 1893 (2007).

[7] O. Maresca et al., J. Chem. Phys. 121, 12548 (2004); G. E. Froudakis, Nano Lett. 1, 531 (2001).

[8] R. T. Yang, Carbon 38, 623 (2000).

[9] L. Duclaux et al., J. Phys. Chem. Solids 67, 1122 (2006).

[10] M. S. Dresselhaus et al., Adv. Phys. 51, 1 (2002).

[11] K. Watanabe et al., Nature Phys. Sci. 233, 160 (1971).

[12] J. P. Beaufils et al., Mol. Phys. 44, 1257 (1981).

[13] A. P. Smith et al., Phys. Rev. B 53, 10187 (1996).

[14] W. J. Stead et al., J. Chem. Soc., Faraday Trans. 2 84, 1655 (1988).

[15] A. Lovell et al., Physica (Amsterdam) 385-386B, 163 (2006).

[16] A. Lovell, Ph.D. thesis, University College London, 2007.

[17] E. P. Gilbert et al., J. Chem. Soc., Faraday Trans. 94, 1861 (1998).

[18] C. J. Carlile and M. A. Adams, Physica (Amsterdam) 182B, 431 (1992).

[19] D. Colognesi et al., Appl. Phys. A 74, S64 (2002).

[20] J. Dawidowski et al., Phys. Rev. B 73, 144203 (2006).

[21] I. P. Silvera, Rev. Mod. Phys. 52, 393 (1980).

[22] Y. Ren et al., Appl. Phys. Lett. 79, 3684 (2001); P. A. Georgiev et al., J. Phys. Condens. Matter 16, L73 (2004).

[23] S. J. Clark et al., Z. Kristallogr. 220, 567 (2005).

[24] D. Vanderbilt, Phys. Rev. B 41, 7892 (1990).

[25] B. R. Johnson, J. Chem. Phys. 67, 4086 (1977).

[26] J. G. Vitillo et al., J. Chem. Phys. 122, 114311 (2005).

[27] H. Margenau and N. R. Kestner, Theory of Intermolecular Forces (Pergamon Press, Oxford, 1971), 2nd ed., p. 17.

[28] S. Patchkovskii et al., Proc. Natl. Acad. Sci. U.S.A. 102, 10439 (2005).

[29] S. Hamel et al., J. Chem. Phys. 121, 12618 (2004).

[30] R. N. Zare, Angular Momentum (Wiley, New York, 1988), Chap. 3.

[31] A. D. McLean et al., J. Chem. Phys. 45, 3676 (1966); W. Kołos et al., J. Chem. Phys. 46, 1426 (1967). 